\title{
Shifting paradigms: whole brain radiation therapy versus stereotactic radiosurgery for brain metastases
}

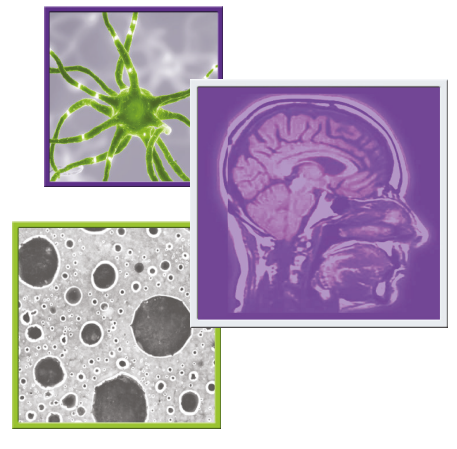

\author{
Ashwin Shinde ${ }^{1}$, David Akhavan ${ }^{1}$, Mina Sedrak², Scott Glaser ${ }^{1}$ \& Arya Amini*,1 \\ ${ }^{1}$ Department of Radiation Oncology, City of Hope National Medical Center, Duarte, CA, 91010, USA \\ ${ }^{2}$ Department of Medical Oncology, City of Hope National Medical Center, Duarte, CA, 91010, USA \\ *Author for correspondence: Tel.: +1 626218 4589; aamini@coh.org \\ "current national guidelines prefer SRS as upfront treatment in patients with 'limited' brain \\ metastases"
}

First draft submitted: 10 December 2018; Accepted for publication: 14 January 2019; Published online: 31 January 2019

Keywords: brain metastases $\bullet$ fractionation $\bullet$ radiation therapy $\bullet$ radiosurgery $\bullet$ stereotactic $\bullet$ whole brain

Management of intracranial metastases with radiation has historically been performed with whole brain radiation therapy (WBRT), which encompasses the entire brain, treating both visible and potentially microscopic disease [1]. In 1961, a Swedish neurosurgeon, Lars Leksell introduced the concept of stereotactic radiosurgery (SRS), in which a high dose of radiation could be delivered to a solitary lesion in a single treatment [2]. The concept of SRS, which has since become standard practice at many institutions, is to deliver higher dose per treatment for better disease control with minimal effect on normal brain tissue, leading ultimately to improved cognitive outcomes. This article will evaluate the shift in intracranial radiation from WBRT to SRS.

\section{SRS for intact brain metastases}

SRS was initially formally evaluated in the Radiation Therapy Oncology Group (RTOG) 90-05 trial as salvage treatment in patients who previously received WBRT, demonstrating safety of single fraction, high-dose radiation [3]. A subsequent RTOG study showed that the addition of SRS to WBRT in patients with one to three brain metastases, with no lesions over $4 \mathrm{~cm}$ in size, improved overall survival (OS) only in patients with a single metastasis [4]. Why a cut-off of three brain metastases was chosen is unclear, but may have been due to evidence of independently worse prognosis in patients with four or more brain metastases [5]. After establishing the safety and efficacy of combination SRS and WBRT, further studies evaluated outcomes when SRS alone was given in the one to three brain metastases population. Two large studies from Europe and Japan comparing SRS alone to SRS and WBRT demonstrated higher rates of intracranial failure rates with SRS alone, without compromising OS outcomes. While WBRT was found to reduce intracranial failure rates, cognitive outcomes were found to be significantly worse, as demonstrated in a randomized study by the MD Anderson group [6-8]. Based on these seminal publications and subsequently others as well, the preferred standard of care for patients with limited intracranial disease, generally considered to be $\leq 3$ metastases with the largest lesion smaller than $4 \mathrm{~cm}$, though the definition of 'limited' continues to evolve, has transitioned to SRS instead of WBRT for most histologies [9].

Initial evidence for use of SRS in patients with higher numbers of brain metastases $(>3)$ came from a Japanese multi-institutional prospective study that enrolled patients with one to ten brain metastases in order to evaluate whether SRS to five to ten brain metastases was noninferior to those with two to four metastases [10]. Eligible patients had a cumulative brain metastasis volume of $\leq 15$ cubic centimeters (cc) and were stratified depending on if they had one, two to four, or five to ten brain metastases. While patients with a single brain metastasis had improved OS, there was no difference in OS between the two to four and five to ten cohorts, suggesting that the previously studied cut-off of three to five metastases may be arbitrary, and perhaps a volume-based approach may be more appropriate for determining eligibility of SRS. A subsequent study has provided more evidence that 
volume of intracranial tumor is more important to prognosis than the number of lesions [11]. There is currently an ongoing randomized Phase III trial comparing WBRT to SRS in patients with four to ten brain metastases (Clinicaltrials.gov\# NCT0235300, [12]). Results from ongoing trials may continue to evolve the definition of 'limited' disease in the brain and the historic answer of three or less may change. Another future trial is comparing WBRT to SRS in patients with five to 15 metastases [13].

\section{Radiation in the postoperative scenario}

Surgical resection is frequently done for brain metastases. Common indications include solitary brain metastasis [14], need for pathological diagnosis, excessively large tumors and/or symptomatology due to mass effect from tumor and/or edema without sufficient response to corticosteroids. Historically, postoperative WBRT was proven to improve rates of brain recurrence and neurological death [15]. A Phase III trial showed that replacing WBRT with SRS as postoperative treatment did not affect survival, but reduced declines in cognitive function [16]. A separate Phase III trial randomizing patients to postoperative SRS or observation again showed no differences in OS but did demonstrate improved rates of local progression with postoperative SRS compared with observation [17]. In summary, postoperative SRS offers a lower risk of cognitive decline compared with WBRT, without sacrificing intracranial local control rates. The optimal timing of radiotherapy following surgery continues to be evaluated.

\section{Fractionation schemes for WBRT \& SRS}

The most standard fractionation scheme for WBRT is 30 Gy in 10 fractions or 37.5 Gy in 15 fractions, although 20 Gy in five fractions can be used for patients with poorer prognosis [9]. For SRS, RTOG 90-05 defined maximum tolerated marginal doses (in patients with previous history of WBRT) of 24, 18 and 15 Gy for tumors $<2,2-3 \mathrm{~cm}$, and $>3 \mathrm{~cm}$, respectively [3]. The most concerning dose-limited toxicity from SRS is radiation necrosis (RN). Multiple studies have identified dose limited constraints for normal brain to reduce the risk of RN. For example, the volume of normal brain receiving $10 \mathrm{~Gy}$ or $12 \mathrm{~Gy}$ in a single fraction of radiation has been predictive of a patient's risk of developing $\mathrm{RN}[18,19]$. In tumors of larger size and volume, patients are at inherently higher risk of developing RN. Given the radiobiological principles of decreased normal tissue toxicity with increasing fractionation, multiple institutional series have demonstrated the feasibility of fractionated SRS, also known as hypofractionated stereotactic radiation therapy (SRT), in an attempt to minimize risks of toxicity. For three-fraction regimens, data have evaluated 7 to 12 Gy per fraction $[19,20]$. The best evidence for a SRT regimen is generally felt to be 9 Gy $\times 3$, per an Italian retrospective study showing improved rates of toxicity and improved tumor control with 9 Gy $\times 3$ SRT compared with single fraction SRS [21]. In regards to a 5-fraction regimen, studies have shown that a total dose of 30 or 35 Gy is reasonable, 40 Gy leads to excessive toxicity, but 25 Gy may have worse oncologic outcomes [22,23]. As a general principle, post-operative treatment with SRS uses equivalent doses to lesions being treated definitively.

\section{Is whole brain radiation dead?}

One of the major concerns with WBRT was the higher incidence of neurocognitive functional deficits. Despite trials showing lower rates of distant intracranial failure with the addition of WBRT to SRS, the corresponding increases in neurocognitive deficits and lack of OS improvement lead most to defer WBRT favoring quality of life. WBRT is still generally indicated in several scenarios including patients with large volume and/or numerous brain metastases. WBRT is also indicated for treatment of brain metastases from small cell lung cancer, both as treatment for visible disease and for prophylactic cranial irradiation. Therefore, interventions to mitigate the risk of neurocognitive deficits were developed and evaluated. Memantine, a N-methyl-D-aspartic acid receptor blocker, was originally used in Alzheimer's dementia patients, but demonstrated neurocognitive protection compared with placebo in patients undergoing WBRT with acceptable toxicity [24]. An additional method developed in the radiation oncology field to mitigate cognitive deterioration with WBRT includes hippocampal sparing or hippocampal avoidance WBRT (HA-WBRT), which, in a Phase II trial, showed better cognitive and equivalent oncologic outcomes compared with historical controls [25]. Most recently, Phase III data comparing WBRT + memantine to HA-WBRT + memantine was presented and showed that HA-WBRT reduced rates of cognitive function failure compared with WBRT, even after both groups of patients received memantine [26]. The authors recommended consideration of HA-WBRT for any patients with WBRT who have an expected survival of 4 months or greater. The authors also made an exploratory statement, that by multiplying the relative benefits of memantine and HA-WBRT compared with WBRT across both trials, rates of neurocognitive decline may be similar to SRS alone. There is one ongoing 
trial comparing WBRT (HA-WBRT will be preferred) to SRS in patients with five to 20 brain metastases, the results of which will be critical to determining whether HA-WBRT and SRS will give similar cognitive outcomes (Clinicaltrials.gov \# NCT03075072).

In conclusion, current national guidelines prefer SRS as upfront treatment in patients with 'limited' brain metastases, defined as a single digit number of lesions (with variable cut-offs depending on radiation oncologist preferences) or total volume of lesions. While WBRT still plays a role in patients with extensive intracranial disease or poor prognosis or performance status, HA-WBRT with memantine may be the olive branch WBRT requires to continue to play a role in the treatment of brain metastases. In regard to the future role of radiation therapy for brain metastases, the field will need to continue to evolve with improvements in targeted and immunologic therapies. How medical and radiation oncologists will combine their modalities together in the future are currently being studied and further research, in the arena of combining targeted therapies and immunotherapy with radiation (either SRS or WBRT) for patients with brain metastases, is necessary.

\section{Financial \& competing interests disclosure}

The authors have no relevant affiliations or financial involvement with any organization or entity with a financial interest in or financial conflict with the subject matter or materials discussed in the manuscript. This includes employment, consultancies, honoraria, stock ownership or options, expert testimony, grants or patents received or pending, or royalties.

No writing assistance was utilized in the production of this manuscript.

\section{Open access}

This work is licensed under theAttribution-NonCommercial-NoDerivatives 4.0 Unported License. To view a copy of this license, visit http://creativecommons.org/licenses/by-nc-nd/4.0/

\section{References}

1. Horton J, Baxter DH, Olson KB. The management of metastases to the brain by irradiation and corticosteroids. Am. J. Roentgenol. Radium Ther. Nucl. Med. 111(2), 334-336 (1971).

2. Leksell L. The stereotaxic method and radiosurgery of the brain. Acta Chir. Scand. 102(4), 316-319 (1951).

3. Shaw E, Scott C, Souhami L et al. Single dose radiosurgical treatment of recurrent previously irradiated primary brain tumors and brain metastases: final report of RTOG protocol 90-05. Int. J. Radiat. Oncol. Biol. Phys. 47(2), 291-298 (2000).

4. Andrews DW, Scott CB, Sperduto PW et al. Whole brain radiation therapy with or without stereotactic radiosurgery boost for patients with one to three brain metastases: Phase III results of the RTOG 9508 randomised trial. Lancet 363(9422), 1665-1672 (2004).

5. Nieder C, Andratschke N, Grosu AL, Molls M. Recursive partitioning analysis (RPA) class does not predict survival in patients with four or more brain metastases. Strablentherapie und Onkol. 179(1), 16-20 (2003).

6. Kocher M, Soffietti R, Abacioglu U et al. Adjuvant whole-brain radiotherapy versus observation after radiosurgery or surgical resection of one to three cerebral metastases: results of the EORTC 22952-26001 study. J. Clin. Oncol. 29(2), 134-141 (2011).

7. Chang EL, Wefel JS, Hess KR et al. Neurocognition in patients with brain metastases treated with radiosurgery or radiosurgery plus whole-brain irradiation: a randomised controlled trial. Lancet Oncol. 10(11), 1037-1044 (2009).

8. Aoyama $\mathrm{H}$, Shirato $\mathrm{H}$, Tago $\mathrm{M}$ et al. Stereotactic radiosurgery plus whole-brain radiation therapy vs stereotactic radiosurgery alone for treatment of brain metastases. JAMA 295(21), 2483 (2006).

9. Bower M, Waxman J. Central nervous system cancers. Nccn Guidel. 1, 10-11 (2016).

10. Yamamoto M, Serizawa T, Shuto T et al. Stereotactic radiosurgery for patients with multiple brain metastases (JLGK0901): a multi-institutional prospective observational study. Lancet. Oncol. 15(4), 387-95 (2014).

11. Kim IK, Starke RM, McRae DA et al. Cumulative volumetric analysis as a key criterion for the treatment of brain metastases. J. Clin. Neurosci. 39, 142-146 (2017).

12. Zindler JD, Bruynzeel AME, Eekers DBP, Hurkmans CW, Swinnen A, Lambin P. Whole brain radiotherapy versus stereotactic radiosurgery for 4-10 brain metastases: a Phase III randomised multicentre trial. BMC Cancer. 17(1), 500 (2017).

13. Roberge D, Brown PD, Whitton A et al. The future is now - prospective study of radiosurgery for more than 4 brain metastases to start in 2018! Front. Oncol. 8, 380 (2018).

14. Patchell RA, Tibbs PA, Walsh JW et al. A randomized trial of surgery in the treatment of single metastases to the brain. N. Engl. J. Med. 322(8), 494-500 (1990).

15. Patchell RA, Tibbs PA, Regine WF et al. Postoperative radiotherapy in the treatment of single metastases to the brain: a randomized trial. JAMA 280(17), 1485-1489 (1998). 
16. Brown PD, Ballman KV, Cerhan JH et al. Postoperative stereotactic radiosurgery compared with whole brain radiotherapy for resected metastatic brain disease (NCCTG N107C/CEC.3): a multicentre, randomised, controlled, Phase 3 trial. Lancet Oncol. 18(8), 1049-1060 (2017).

17. Mahajan A, Ahmed S, McAleer MF et al. Post-operative stereotactic radiosurgery versus observation for completely resected brain metastases: a single-centre, randomised, controlled, Phase 3 trial. Lancet Oncol. 18(8), 1040-1048 (2017).

18. Blonigen BJ, Steinmetz RD, Levin L, Lamba MA, Warnick RE, Breneman JC. Irradiated volume as a predictor of brain radionecrosis after linear accelerator stereotactic radiosurgery. Int. J. Radiat. Oncol. 77(4), 996-1001 (2010).

19. Minniti G, Clarke E, Lanzetta G et al. Stereotactic radiosurgery for brain metastases: analysis of outcome and risk of brain radionecrosis. Radiat. Oncol. 6, 48 (2011).

20. Eaton BR, Gebhardt B, Prabhu R, Shu H-K, Curran WJ, Crocker I. Hypofractionated radiosurgery for intact or resected brain metastases: defining the optimal dose and fractionation. Radiat. Oncol. 8(1), 135 (2013).

21. Minniti G, Scaringi C, Paolini S et al. Single-fraction versus multifraction $(3 \times 9$ Gy) Stereotactic Radiosurgery for Large $(2 \mathrm{~cm})$ brain metastases: a comparative analysis of local control and risk of radiation-induced brain necrosis. Int. J. Radiat. Oncol. 95(4), 1142-1148 (2016).

22. Lischalk JW, Oermann E, Collins SP et al. Five-fraction stereotactic radiosurgery (SRS) for single inoperable high-risk non-small cell lung cancer (NSCLC) brain metastases. Radiat. Oncol. 10, 216 (2015).

23. Marcrom SR, McDonald AM, Thompson JW et al. Fractionated stereotactic radiation therapy for intact brain metastases. Adv. Radiat. Oncol. 2(4), 564-571 (2017).

24. Brown PD, Pugh S, Laack NN et al. Memantine for the prevention of cognitive dysfunction in patients receiving whole-brain radiotherapy: a randomized, double-blind, placebo-controlled trial. Neuro. Oncol. 15(10), 1429-1437 (2013).

25. Gondi V, Pugh SL, Tome WA et al. Preservation of memory with conformal avoidance of the hippocampal neural stem-cell compartment during whole-brain radiotherapy for brain metastases (RTOG 0933): a Phase II multi-institutional trial. J. Clin. Oncol. 32(34), 3810-6 (2014).

26. Gondi V, Pugh S, D Brown P et al. NCOG-01. Preservation of neurocognitive function (NCF) with hippocampal avoidance during whole-brain radiotherapy (WBRT) for brain metastases: preliminary results of Phase III trial NRG oncology CC001. Neuro. Oncol. 20(suppl_6), vi172-vi172 (2018). 